examples of an ESE associated with disease: spinal muscular atrophy (SMA), a paediatric neurodegenerative disorder caused by the loss of both functional copies of the survival of motor neuron 1 (SMN1) gene. In this condition, the loss of SMN1 protein could be compensated by a related gene product called SMN2, but a single-nucleotide mutation in exon 7 of SMN2 produces an isoform that lacks this exon, resulting in a defective protein. The authors showed that creating an ESSENCE compound that targets this mutation restored the inclusion of exon 7 in the transcript in vitro. The technique is being developed to optimize in vivo delivery and activity, with the hope that the next generation of ESSENCE compounds will represent a viable approach for the treatment of SMA and many other genetic diseases.

Simon Frantz

(Q) References and links ORIGINAL RESEARCH PAPER Cartegni, L. \& Krainer, A. R. Correction of disease-associated exon skipping by synthetic exon-specific activators. Nature Struct. Biol. 10, 120-125 (2003) FURTHER READING Cartegni, L., Chew, S. L. \& Krainer, A. R. Listening to silence and understanding nonsense: exonic mutations that affect splicing. Nature Rev. Genet. 3, 285-298 (2002)

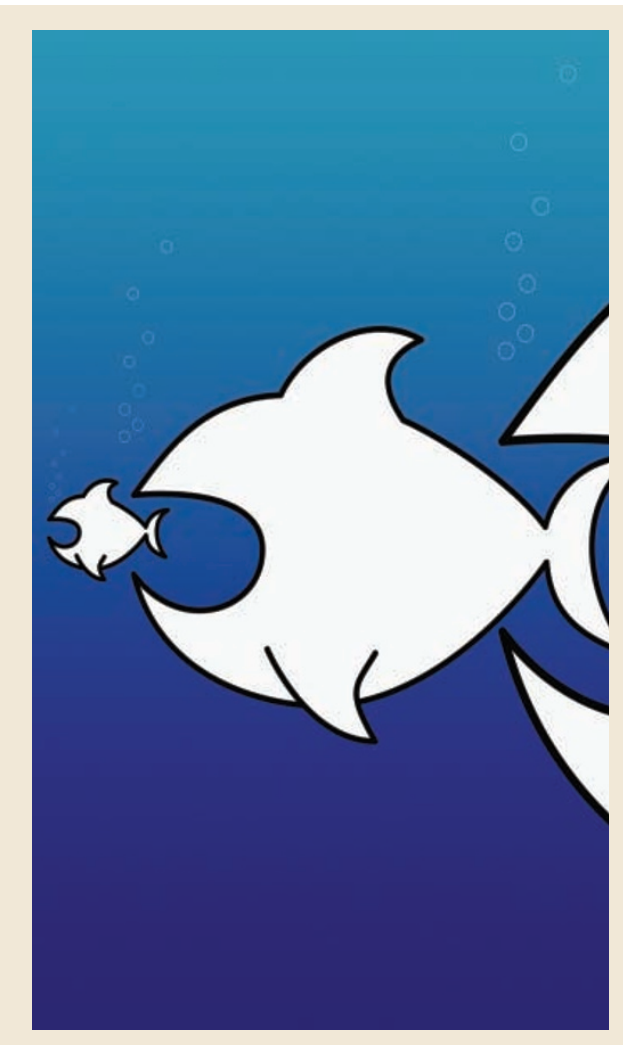

\section{STRUCTURE-BASED DRUG DESIGN}

\section{Can GPCR models work?}

Without doubt, the determination of the crystal structure of the G-protein-coupled receptor (GPCR) rhodopsin in 2000 was a landmark in the study of GPCRs. However, given the considerable challenges inherent in repeating this feat with other GPCRs, the key question for those involved in GPCR-based drug discovery is how well this structural information can be extrapolated to other GPCRs of therapeutic interest. As described in Proteins, Rognan and colleagues have tackled this issue, and show that models of a range of GPCRs based on the rhodopsin structure can be successfully used to identify both antagonists and agonists by virtual screening of compound libraries.

First, the authors constructed homology-based models of three well-studied GPCRs - the dopamine $\mathrm{D}_{3}$ receptor, the acetylcholine muscarinic receptor and the vasopressin $V_{1 a}$ receptor - by alignment with the rhodopsin sequence, mutation of the appropriate amino acids and then 'energy minimization' as a first step to optimize the protein conformation. To better model the potential 'antagonist-bound' state for each GPCR, a known antagonist was 'docked' into the active site using experimental binding data, and a further energy minimization performed.

Once the known antagonist was removed, the models were ready for testing. In each case, 10 known antagonists (but not those used in the previous energy minimization) were added to 990 drug-like compounds to give a virtual library of 1,000 compounds for each GPCR. These compounds were then docked into the appropriate GPCR model and their potential for binding assessed computationally. In all three cases, the procedure could successfully identify the known antagonists. For example, for the $\mathrm{D}_{3}$ receptor the optimum hit list of 37 potential binders contained seven of the known antagonists - a hit rate of $20 \%$, or 20 -fold higher than random screening of the full library would give ( 10 true hits out of 1,000 compounds). Importantly, an analysis of the structures of the ligands in the hit lists indicated that they were not biased by the antagonist used in the energy-minimization step, which means that it should be possible to retrieve new lead structures unrelated to any known GPCR ligand.

But would the procedure prove equally successful for modelling the 'agonist-bound' activated receptor state, which differs significantly from the 'antagonist-bound' receptor ground state, both in overall conformation and degree of

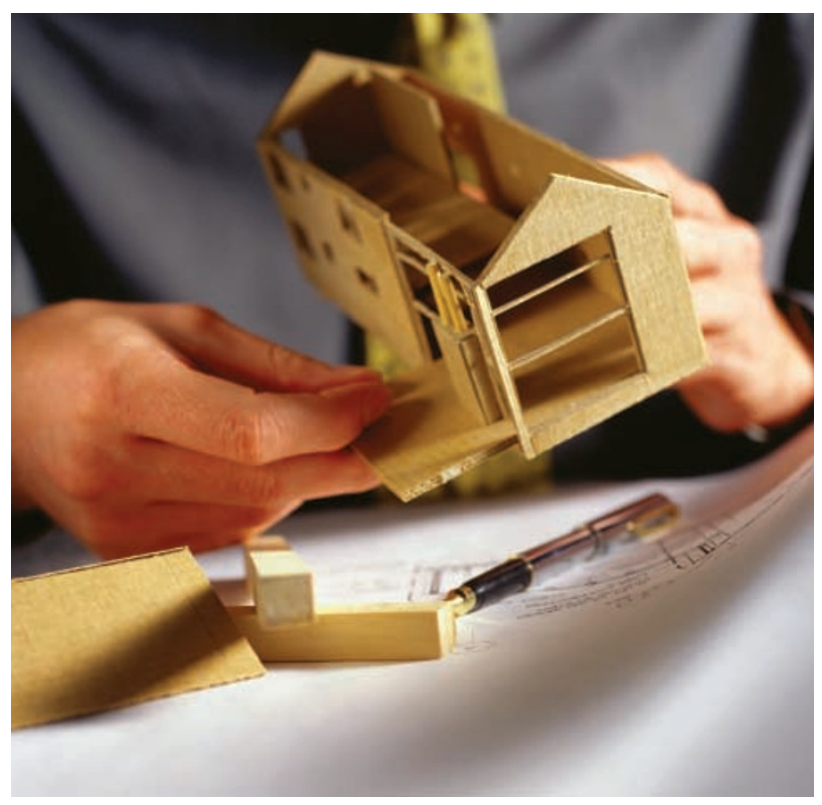

flexibility? After first making manual alterations to the GPCR structures assessed - the $\mathrm{D}_{3}$ receptor, the $\beta_{2}$ adrenoceptor and the $\delta$-opioid receptor to reflect the major conformational changes known to occur on receptor activation, the authors repeated the procedure used for antagonists, but also assessed a modified procedure to reflect the fact that there could be more than one 'activated' state for each receptor. Rather than just using one agonist to model the agonist-bound state, several different agonists were superimposed to generate a 'pharmacophore' model that should better represent the most important structural features of different agonist classes for the target receptors. Such pharmacophore models were then used in the energy minimization step, as before, to give a model of the agonist-bound state of each receptor.

An assessment of the hit lists after virtual screening of the 1,000-compound library for each receptor revealed that the pharmacophore-based models were significantly more successful than than those created by just single-agonist minimization, with hit rates comparable to those found with antagonists. For example, for the $\mathrm{D}_{3}$ receptor a hit rate of $30 \%$ was achieved, and again hits did not seem biased towards the ligand structures used in the generation of the receptor model. So, it seems that such approaches could be usefully exploited in searches for novel GPCR agonists and antagonists (once enough information is available on true ligands to allow model building), perhaps most profitably as an initial 'filtering' step to select the most promising compounds for experimental high-throughput screening.

Peter Kirkpatrick

(1) References and links

ORIGINAL RESEARCH PAPER Bissantz, C., Bernard, P., Hibert, M. \& Rognan, D. Protein-based virtual screening of chemical databases. II. Are homology models of G-protein coupled receptors suitable targets? Proteins 50, 5-25 (2003) 